\title{
Beyond-Line-of-Sight Communications with Ducting Layer
}

\author{
Ergin Dinc, Student Member, IEEE, Ozgur B. Akan, Senior Member, IEEE
}

\begin{abstract}
Near-surface wave propagation at microwave frequencies especially $2 \mathrm{GHz}$ and above shows significant dependence on atmospheric ducts that are the layer in which rapid decrease in the refractive index occurs. The propagating signals in the atmospheric ducts are trapped between the ducting layer and the sea surface, so that the power of the propagating signals do not spread isotropically through the atmosphere. As a result, these signals have low path-loss and can travel over-the-horizon. Since atmospheric ducts are nearly permanent at maritime and coastal environments, ducting layer communication is a promising method for beyond-Line-of-Sight (b-LoS) communications especially in naval communications. To this end, we overview the characteristics and the channel modeling approaches for the ducting layer communications by outlining possible open research areas. In addition, we review the possible utilization of the ducting layer in Network Centric Operations (NCO) to empower decision making for the b-LoS operations.
\end{abstract}

Index Terms-Atmospheric ducts, ducting layer, refractivity, beyond-line-of-sight communications

\section{INTRODUCTION}

A TMOSPHERIC ducts that are caused by rapid decrease in the refractive index of the lower atmosphere have tremendous effects on the near-surface wave propagation. In the presence of atmospheric ducts, the signals below the atmospheric duct are trapped between the sea surface and the duct. Therefore, the signals do not spread isotropically through the atmosphere. Instead, the signals spread mostly in the ducting layer which is the area between the sea surface and the duct as in Fig. 1. As a result, the spreading loss decreases considerably compared to the standard atmosphere and there is a significant amount of increase in the received signal power. In this way, the trapped signals can travel overthe-horizon and it makes the ducting layer convenient for bLoS communications.

The formation of atmospheric ducts entirely depends on the refractivity of the lower atmosphere that is determined by atmospheric parameters such as wind, pressure, temperature and most importantly humidity that is the key and essential factor in the duct formation as will be further discussed in Section II [1]. Experimental studies also show that atmospheric ducts are nearly permanent in the coastal and maritime environments due to high evaporation rates. As a result, the ducting layer communication becomes the dominant propagation mechanism at the lower troposphere especially between 2 to $20 \mathrm{GHz}$. Since the transmitter and the receiver antenna should be located within the ducting layer and this mechanism is effective at the lower troposphere, especially naval communications can utilize b-LoS communications with the ducting layer.

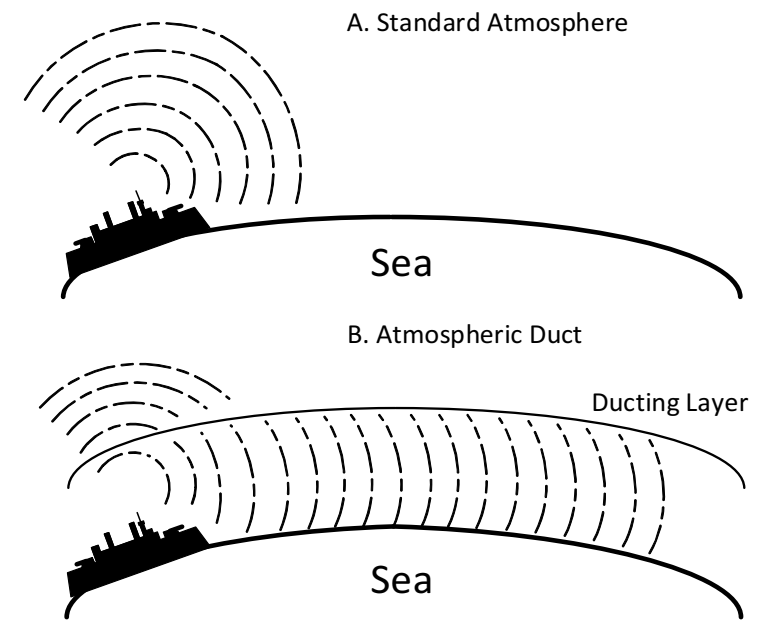

Fig. 1. Signal spreading in the standard atmosphere and the atmospheric duct.

Ducting layer studies mainly focus on refractivity estimation techniques and radar path-loss calculations [1], [2]. However, there are a few studies which focus on the utilization of the atmospheric ducts as a communication medium. To this end, [3] provides a high data rate employment of the evaporation ducts for b-LoS sensor networks where continuous satellite communication is expensive and high data rate cellular communication is unavailable. In [3], the ducting layer is utilized to monitor a reef site in Great Barrier Reef Australia. According to their results, the atmospheric ducts can connect a $78 \mathrm{~km}$ link at $10.5 \mathrm{GHz}$ with $10 \mathrm{Mbps}$ at $80 \%$ of the time. Based on the available experimental results and our reviews, especially state-of-the-art military systems can connect distances up to $500-1000 \mathrm{~km}$ with the ducting layer.

Modern naval b-LoS communications can utilize either HF (high frequency) radio systems that are band-limited to support high data rates, or satellites that are prone to hostile jamming and have high transmission delays, or relay nodes that can be exposed to hostile attacks. There is a significant need for a direct transmission channel between b-LoS units to empower strategical and tactical decisions in NCO. To this end, the ducting layer is a promising communication medium for bLoS applications because atmospheric ducts can provide a direct communication channel between navy-navy and navylow flying units (especially unmanned aerial vehicle (UAVs)) thanks to their low spreading loss. In this way, a naval ship can relay the information, that is coming from LOS sources, to a b-LoS unit within the ducting layer. Thus, ducting layer 
can provide a b-LoS link between sites with low transmission delays and low probability of detect and intercept [4].

The main contribution of this work will be the overview of the characteristics of the ducting layer b-LoS communications and the possible utilization of the ducting layer in the b-LoS naval communication networks. To this end, we review the channel modeling techniques and the software tools that can be used for the ducting layer. We present a preliminary analysis to show that ducting layer can achieve lower path-loss up to $20 \mathrm{~dB}$ at $500 \mathrm{~km}$ compared to the free space. In addition, we make a linear regression fitting for the path loss curve to find the path-loss exponent of the channel. To the best of our knowledge, there is no study which considers the path-loss exponent for the ducting layer. Therefore, this study provides both the characteristics of the ducting layer and the possible application areas by outlining the open research issues in the field.

The remaining of the paper is organized as follows. Section II provides background information about the refractivity and different types of atmospheric ducts. In addition, the effects of ducts on wireless communication are discussed at the end of the section. In Section III, the channel modeling approaches and the software tools are reviewed, and a preliminary analysis which investigates the path-loss exponent of the ducting layer is presented. Furthermore in Section III, the open research issues on the ducting layer channel modeling are also pointed out. Lastly, the possible application areas of ducting layer bLoS communications can be found in Section IV.

\section{Properties of Atmospheric Ducts}

The formation and the effects of atmospheric ducts entirely depend on the refractivity of the lower atmosphere. This section provides background for the refractivity, formation of ducts and effects of ducts on wireless communications.

\section{A. Refractive Index, Refractivity and Modified Refractivity}

Tropospheric radio refractive index $(n)$ relies on weather conditions: wind, temperature, pressure and humidity at the lower atmosphere. Refractive index changes slightly with height. It varies between 1.000250 and 1.000400. Therefore, instead of the tropospheric radio refractive index, refractivity, which is the scaled version of the tropospheric radio refractive index, will be used and the refractivity $(N)$ is defined as [5]

$$
N=(n-1) \times 10^{6} \mathrm{~N} \text {-units, }
$$

where $n$ is the atmospheric refractive index.

(1) is an idealized refractivity expression in which the earth surface is assumed to be flat, but to be more realistic the earth's curvature and the variation of height are considered in the modified refractivity $(M)$ [5]

$$
\begin{aligned}
M & =N+\left(\frac{h}{a}\right) \times 10^{6} \\
& =N+157 \times h \text { M-units }
\end{aligned}
$$

where $h$ is the height above sea level in $\mathrm{km}$ and $a$ is the radius of the earth in $\mathrm{km}$.
Tropospheric wave propagation depends on the rate of change in the modified refractivity that is associated with the refractivity gradient $(\partial M / \partial h)$. There are four different refractive conditions according to the refractivity gradient as in Fig. 2. In sub-refraction condition, the signal is bent away from the surface. Standard refractivity condition is associated with the wave propagation in the standard atmosphere. Superrefraction condition causes the signal to propagate downward at a rate which is larger than the standard condition and smaller than the curvature of the earth.

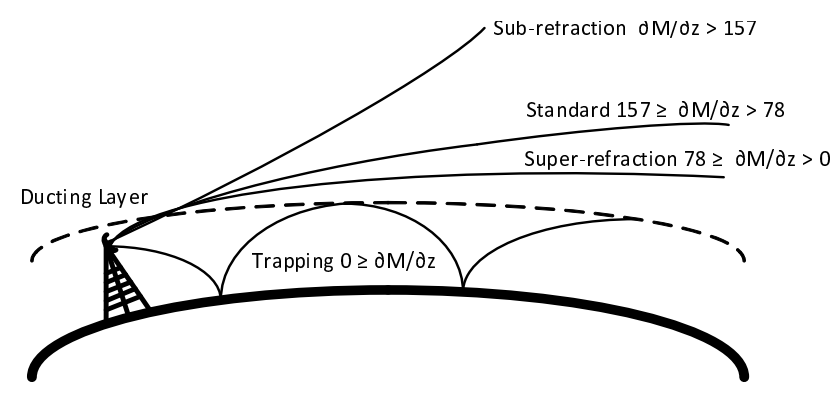

Fig. 2. Rays under various refractive conditions.

The last and most important one is the trapping condition in which the signal is refracted back to the earth's surface. In this way, the refracted rays are trapped between surface or sea and ducting layer. The trapping condition is associated with a negative modified refractivity gradient and duct height is given by the height where the gradient of the modified refractivity changes from negative to positive, see Fig. 3.

The refractivity gradient and the duct height are the most important parameters for modeling atmospheric ducts. The characteristics of atmospheric ducts can be determined via the atmospheric parameters by using radiosonde or bulk atmospheric measurements. In addition, indirect methods are also available such as refractivity from clutter techniques which estimates the refractivity of the lower atmosphere from the clutter signal returns [2].

\section{B. Formation and Characteristics of Ducts}

The duct formation is an atmospheric phenomenon. Humidity, air-sea temperature, pressure and wind play significant roles in the duct formation. Among all the atmospheric conditions, humidity is the key and essential factor in the process. As a result, duct formation is more probable in regions with high evaporation rates such as equatorial and tropical regions.

The rapid humidity decrease in the few meters of the lower troposphere causes the duct formation. In addition, air-sea temperature is the most important factor which contributes to the process. When the air is warmer than the earth or sea surface, the temperature inversion occurs and it enhances the duct formation. Temperature inversion is caused by advection of dry and warm air mass or cooling of land when the air is still warm at nights. In addition, negative air-sea temperature difference can prevent the duct formation. This weather condition is named as unstable condition. Factors such as rough terrain, high winds, cold, stormy, rainy, and cloudy conditions also have destructive effects on the duct formation [1]. 
According to their physical formation process, there are three main types of atmospheric ducts:

1) Evaporation duct: Humidity difference in the air-sea boundary causes the formation of evaporation ducts. High evaporation rates are essential for evaporation ducts. Evaporation duct is the most common type of duct formation and it presents up to $90 \%$ of the time in equatorial and tropical regions. The $M$ profile of the evaporation duct is modeled with the logarithmic curve in Fig. 3(a) [6]. Evaporation duct height is mostly between $10-20 \mathrm{~m}$ and it rarely reaches up to $40 \mathrm{~m}$.

Evaporation ducts are the most promising duct formation which have potential to be utilized in the wireless communication applications thanks to their high availability rates. Since evaporation ducts are nearly permanent at coastal and maritime environments, b-LoS communications with the evaporation ducts can be employed especially for naval communications.

2) Surface-based duct: Surface-based ducts are mostly caused by advection of warm and dry air over the ocean or land which creates humidity and temperature inversion. Surfacebased ducts can be modeled with trilinear curve in which the negative slopped part represents the trapping layer as in Fig. 3(b) [2] and the duct height can reach hundreds of meters. Surface-based ducts occur up to $40 \%$ of the time depending on the season and location and these statistics can be found in [7].

3) Elevated duct: The presence of marine layers results in the formation of elevated ducts. The physical mechanism that creates elevated ducts is similar with surface-based ducts. However, in the elevated ducts the lower boundary of the trapping layer is above the surface. Elevated ducts occur at relatively higher heights between $600 \mathrm{~m}$ up to $3000 \mathrm{~m}$ and can also be modeled with trilinear curve in Fig. 3(c) [2]. Especially, air-to-ground links are prone to the trapping effect of the elevated ducts because trapped signals follow longer paths than expected and can reach to the receiver with higher delay spreads and different angles than expected. Therefore, elevated ducts have potential to create unexpected interference in the air-to-ground links. The occurrence rate of the elevated ducts can be up to $50 \%$ according to season and location, and these statistics can be also found in [7].

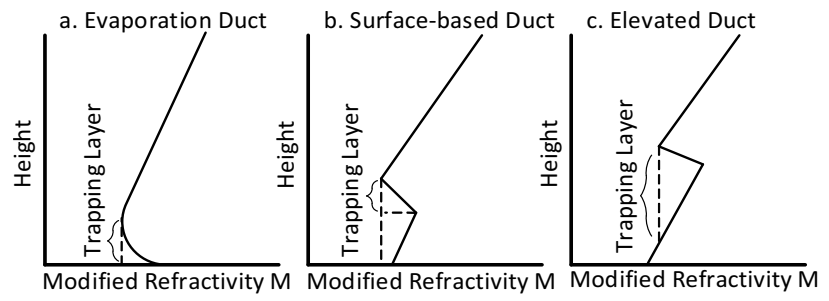

Fig. 3. Modified refractivity models for duct types.

\section{Effects of Ducts on Wave Propagation}

Besides the path loss enhancements, atmospheric ducts also introduce some challenges in the b-LoS wireless communications. Main challenges introduced by the atmospheric ducts and the techniques to overcome these challenges can be outlined as follows:

1) Inter-Symbol-Interference (ISI) : Atmospheric ducts trap the propagating signals at the lower atmosphere and causes signals to follow different paths. The trapped signals reach the receiver with different delay spreads and phase components. Therefore, these trapped signals can create destructive/constructive interference at the receiver. The receiver should optimally combine these components by using adaptive antenna techniques to prevent interference. To this end, optimal transmit and receive beam patterns should be designed according to the characteristics of the atmospheric ducts and these techniques provides both diversity and array gain along with interference suppression as recommended in [4].

2) Unexpected interference: Up to now, we consider both transmitter and receiver are located within the duct. On the other hand, the effects of ducts are prominent even if one of the antennas stay within the duct such as satellite communications and air-to-ground links in maritime and coastal environments. When satellite signals have low elevation angles, these signals can be trapped by atmospheric ducts and the trapped signals may be received by the receiver with different delay spreads and angle-of-arrivals (AOA). As a result, the receiver may misinterpret AOA of the incoming signal which is refracted by the ducting layer, and the surveillance system can be adversely affected by this effect.

The trapped signals in ducts follow longer paths and reaches to the receiver with higher delays as experimentally measured in [8]. Therefore, even in LOS ranges, this effect can create multipath fading and change the pattern of the interference in naval systems. As a result, (OFDM) can be promising for the ducting layer if the channel creates high delay spreads that makes the coherence bandwidth much lower than the bandwidth of the signal, orthogonal-frequencydivision-multiplexing

3) Sea surface roughness: The trapped signals in the ducting layer are in contact with the sea surface. Especially under strong winds, open sea surfaces form rough surface boundaries. As a result, the reflection from rough sea surface makes signals follow different paths and even some of the transmitted signals are backscattered. Therefore, the sea surface roughness causes decrease in the received power strength and modeling of rough surface boundary is an important challenge for the ducting layer propagation modeling. There are some experimental studies that investigate the effects of rough sea surfaces on the wave propagation within ducts [9], but these experimental works do not provide enough data to model extreme surface conditions and there are hardly a few theoretical models for the sea surface roughness at the presence of atmospheric ducts. To this end, this topic requires both experimental and theoretical research attention.

4) Aerosols and weather conditions: The presence of aerosols such as bacteria, pollen and dust are highly probable in the lower atmosphere. The presence of such aerosols in the communication layer may create scattering and reflection which cause radar echoes and path-loss increase at the receiver side. In addition, hydro-meteors in the lower atmosphere, especially rain, can create significant path-loss due to its 
reflective effects between 10-20 GHz. To this end, rain and aerosol attenuation in the ducting layer is a promising open research field as well.

\section{Channel Modeling Techniques for ATMOSPHERIC DUCTS}

Ducting layer wave propagation is complex due to the trapping effect because the trapped signals are in contact with both rough sea surface and duct layer. In addition to these factors, the ducting layer wave propagation depends on multiple channel parameters such as, antenna heights, duct height and type, carrier frequency and polarization. In this section, we are going to review the channel model approaches to model the ducting layer communication by outlining open research fields. More importantly, we present preliminary simulation results for analyzing the path-loss exponent to indicate the received signal level improvements:

\section{A. Parabolic Equation Methods}

Parabolic equation (PE) method utilizes parabolic approximation to Helmholtz wave equation. This technique is the dominant technique to model the ducting layer wave propagation with the capabilities of including both complex boundary conditions and refractivity variations of the lower atmosphere [10]. The existing numerical methods can provide fast and accurate results for PE. For these reasons, PE methods can be used especially for estimating the large scale path-loss in the ducting layer under different refractivity condition, polarization and surface types.

The remaining of the section includes the numerical methods to solve PE, available software tools and preliminary simulation results to show the signal level enhancement due to the atmospheric duct formation.

1) Numerical Methods for PE: PE can be solved by numerical operations and there are three popular numerical methods for solving PE: Split Step Fourier (SSF), Finite Difference (FD) and Finite Element (FE) [11]. The choice between these methods strongly depends on the scenario and conditions. The SSF method takes advantage of FFT algorithms. Therefore, the implementation of PE with SSF is computationally efficient compared to other methods and, the technique can yield accurate and stable solutions. Secondly, FD provides highest resolution in modeling the boundary conditions by utilizing Crank-Nicholson finite difference scheme. Lastly, FE provides better modeling of the fast variations in the atmospheric conditions and provides flexibility to model the complex boundary conditions. A good review of these techniques can be found in [11].

2) Wave Propagation Tools: There are available wave propagation tools that can be used to model the ducting layer. The most important and widely used one is AREPS that is developed by the Atmospheric Propagation Branch at the Space and Naval Warfare Systems Center, San Diego [12]. AREPS is a hybrid wave propagation tool that combines ray optics and PE with SSF methods. It is capable of taking refractivity index as input from various sources like satellite, meteorological and bulk measurements. In addition, PETOOL, that is a free and on-line available tool, can model both forward and backward scattered waves under different terrain and refractivity condition [13]. Since PETOOL also considers the backward scatter waves unlike AREPS, it can be used to analyze regions with terrain block between the transmitter and the receiver such as islands. PETOOL is calibrated with using ray based methods and AREPS as described in [13]. Since, PETOOL can give reliable results like AREPS and it is a free on-line available tool, researchers may prefer PETOOL to model the wave propagation in the ducting layer. Furthermore, TEMPER, that is developed by the John Hopkins University Applied Physics Laboratory, is especially preferred for the R\&D applications such as high fidelity radar design and 3D wave modeling applications. TEMPER is solely based on PE method [14]. Lastly, Wireless InSite, which is developed by REMCOM, is a hybrid model and it utilizes FD time domain method in order to solve PE. Although, this tool is not widely used in the ducting layer wave propagation modeling, it is a promising modeling tool for the projects that require modeling of complex boundary conditions.

3) Path-loss Analysis for Evaporation Duct: In this section, we present our preliminary analysis to show the received power enhancement by the atmospheric ducts. Since evaporation ducts are more probable compared to other types of ducts, we analyze an ideal $13 \mathrm{~m}$ evaporation duct which is modeled with a logarithmic curve as in [6]. We utilize PETOOL to solve PE for our preliminary large scale path-loss analysis.

Large scale path-loss in wireless communication can be represented with the generic formula as:

$$
P L=A+10 \gamma \log d / d_{0}+X_{s}
$$

where $\gamma$ is the path-loss exponent, $d_{0}$ is the b-LoS range, $A$ is the path-loss at $d_{0}$ and $X_{s}$ is the shadow fading.

Ducting conditions show significant dependence on the carrier frequency because the trapping condition becomes dominant above a certain frequency [5]. To this end, we simulate the wave propagation within a $13 \mathrm{~m}$ evaporation duct for different carrier frequencies (Transmitter is $4 \mathrm{~m}$ above sea level). Fig. 4(a) represents the receiver height vs. path-loss at $80 \mathrm{~km}$ distance. As noticed from this figure, $10.5 \mathrm{GHz}$ can reach the lowest path-loss values and similar experimentally validated results can be found in [3]. Therefore, the signal level enhancements becomes highest in this high frequency range.

To show the signal level enhancement with respect to the free space, we present path-loss vs. range at $10.5 \mathrm{GHz}$ for the transmitter and the receiver that are located at $4 \mathrm{~m}$ above sea level. As in Fig. 4(b), path-loss in the ducting layer is significantly lower than the free space. Therefore, we can expect the path-loss exponent to be smaller than 2 (free space) because the signal energy is not spread through the atmosphere. Instead, it is concentrated within the ducting layer. The simple linear regression analysis with (3) yields the path-loss exponent of 1.2087 for ideal duct and flat sea surface (Black line in Fig. 4(b)). We expect that the path-loss exponent will be higher for the real world conditions with range dependent refractivity conditions and rough sea surface. 


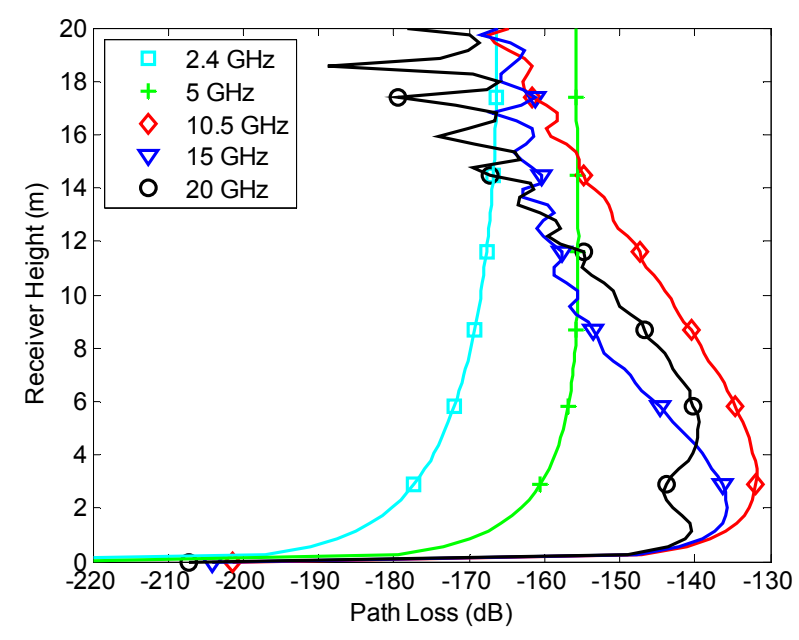

(a) Receiver height vs. path-loss.

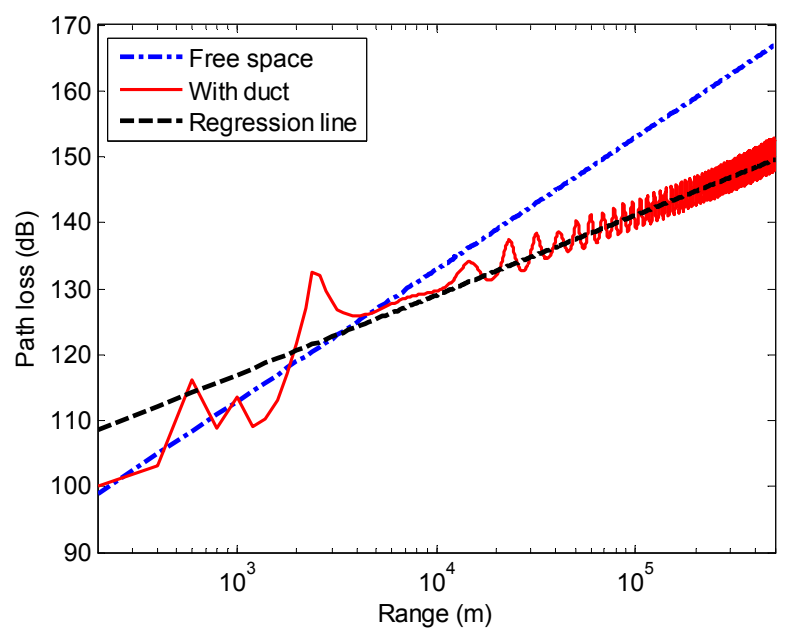

(b) Path-loss vs. range.

Fig. 4. Path-loss variations with respect to receiver height and range in $13 \mathrm{~m}$ ideal evaporation duct.

Although several works are available to model the amount of path-loss within the atmospheric ducts, there are no studies that provides the statistical modeling of the path-loss exponent and the shadow fading with respect to the atmospheric conditions, sea surface roughness, duct height, antenna heights and carrier frequency in the ducting layer communication. In addition, the effects of the polarization and the correlation between antennas are the open research fields which can be solved by PE simulations.

\section{B. Ray Optics Methods}

Ducting layer wave propagation modeling is dominated by PE because the ray analysis introduce significant errors at high ranges and they are much slower. However, the ray optics methods are still used in the hybrid models in order to provide fast and reliable estimates of the behavior of the wave-front and propagation angles [10].

The ray analysis utilize the solution for the Eikonel equation under nonstandard refractivity conditions and they can give the approximate behavior of the delay spreads and AOA of the multipath components. Since PE is inefficient to model these parameters which are essential to the channel modeling, the ray optics methods should be used in the ducting layer studies. There are some experimental studies that focus on AOA at the presence of the atmospheric ducts [15]. In [15], they also estimate AOA for the ducting layer by using both ray optics and PE methods. However, the distribution of delay spreads and $\mathrm{AOA}$ in the ducting layer are not well studied fields, and they require significant experimental and theoretical research efforts. In addition, angle-of-departures (AOD) should be also studied for the ducting layer. Since only signals having low grazing angles can be trapped by the ducting layer, most of the transmitted power can spread through the atmosphere instead of the ducting layer if the beamwidth of the antenna exceeds a certain value which depends on the antenna placement and the atmospheric conditions. To prevent signal spreading through the atmosphere instead of the ducting layer, the maximum beamwidth that can be trapped by the atmospheric ducts should be derived with respect to transmitter antenna height, duct height and carrier frequency by utilizing the ray optics methods.

\section{Applications Areas of the Ducting Layer B-LOS COMMUNICATION}

This section provides answers for "How the usage of the atmospheric ducts as a propagation medium can improve current state-of-art naval communication links?". To this end, we review the possible application areas of the ducting layer b-LoS communications by especially focusing on the military naval applications.

1) Military applications: In current naval b-LoS communications, the transmitter and the receiver can use HF (high frequency) radio systems, or satellites or air units as a relay node to deliver data packages. However, HF radio systems are band-limited in their nature and they are inadequate to provide high data rates that modern military communications require. The other highly used option is the satellite communication which is expensive and introduces high communication delays. In addition, the satellite communication has security problems in the modern warfare because it can be highly affected by hostile jamming. Furthermore, using an air unit as a relay node is dangerous because the unit can be exposed to hostile attacks and the replacement of a relay node can waste considerable amount of mission time [4]. To this end, the ducting layer b-LoS communications is a promising alternative because the communication at the lower troposphere is less prone to be affected by hostile jamming and also, the utilization of the ducting layer eliminates the need for an air unit as relay node. The ducting layer is expected to employ near $10 \mathrm{GHz}$ as in Section III, and with the signal level enhancements, it will be able to provide high data rates that modern military applications require.

In NCO, the connectivity between units are critical to empower strategical and tactical decisions. With the employ- 
ment of the atmospheric ducts, there will be a direct communication channel between navy-navy and navy-low flying units (especially UAVs). Therefore, the employment of ad hoc networking with the b-LoS ducting layer can provide endto-end connectivity between naval warfare. For example, a naval ship can relay an information that is coming from a LOS source to a b-LoS naval ship. To this end, the ad hoc bLoS networks with the atmospheric ducts should be designed and, the distribution of data rates and relay delays should be derived for the ducting layer b-LoS systems. Since there are hardly a few works which uses the atmospheric ducts as a communication medium, the ad hoc networking with the ducting layer is a promising open research field to improve the naval NCO.

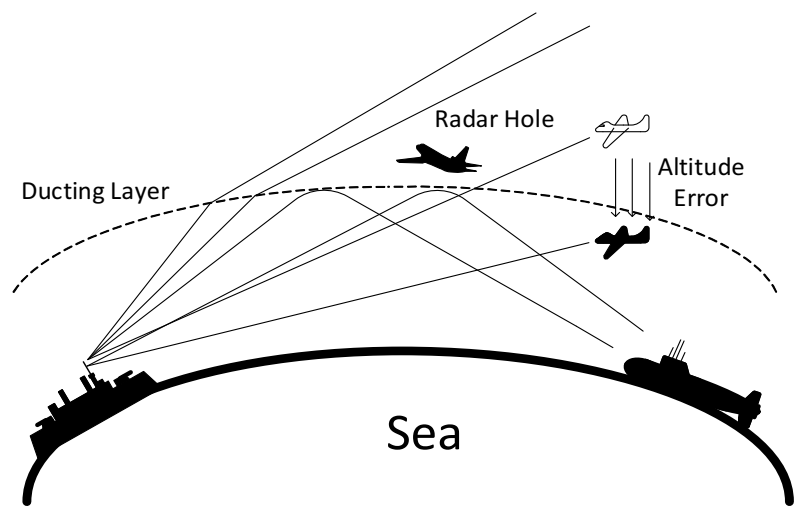

Fig. 5. Wave Propagation with ducting layer.

2) Radar applications: Radar coverage is strongly affected by ducts in coastal and maritime environments [2]. Without considering the effects of ducts, the coverage can be miscalculated and miscalculations impose unwanted problems like radar holes and altitude errors as shown in Fig. 5. Since the military radar applications require precise and accurate estimations, the effects of ducts should be taken into account in the coverage estimations. Therefore, the ducting layer studies also contribute to the radar coverage analysis as well.

3) Wireless Sensor Networks: Ducting layer b-LoS communications can be used to monitor civil or military sensor networks in maritime and coastal areas as in [3] because the implementation of satellite or cellular communication are expensive in large maritime areas. As a result, the ducting layer can provide low cost high data rate monitoring for the maritime wireless sensor networks.

\section{CONClusion}

Ducting layer b-LoS communication is a promising communication method especially for the military naval applications to empower tactical decisions in NCO. Since the ducting layer b-LoS communications show strong dependence on the refractive index which is highly affected by the atmospheric conditions, well-developed channel models are required to determine the characteristics of the channel. To this end, this paper review the channel modeling approaches by outlining the open research areas, and the possible application areas of the ducting layer b-LoS systems. More importantly, this work also provides the path-loss exponent for an ideal evaporation duct.

\section{REFERENCES}

[1] C. Yardim, Statistical estimation and tracking of refractivity from radar clutter, Ph.D. dissertation, Univ. California, San Diego, CA, Mar. 2007.

[2] H. V. Hitney, J. H. Richter, R. A. Pappert, K. D. Anderson, G. B. Jr. Baumgartner, "Tropospheric radio propagation assessment," Proceedings of the IEEE, vol. 73, no. 2, Feb. 1985, pp. 265-283.

[3] G. S. Woods, A. Ruxton, C. Huddlestone-Holmes, G. Gigan, "HighCapacity, Long-Range, Over Ocean Microwave Link Using the Evaporation Duct," IEEE J. Oceanic Eng., vol. 34, no. 3, Jul. 2009, pp. 323-330.

[4] M. J. Luddy, J. H. Winters, A. Lackpour. (2011). Beyond Line-ofSight Communications with Smart Antennas (BLoSSA) [online]. Available:http://www.atl.lmco.com/papers/1978.pdf.

[5] B. R. Bean, E. J. Dutton, Radio meteorology, Dover Publications, New York, 1966.

[6] R. A. Paulus, "Evaporation duct effects on sea clutter," IEEE Transactions on Antennas and Propagation, vol. 38, no. 11, Nov. 1990, pp. 1765-1771.

[7] "ITU-Rs Rec. P. 453-10: The radio refractive index: its formula and refractivity data," ITU, Feb. 2012.

[8] Yu Song Meng, Yee-Hui Lee, "Measurements and Characterizations of Air-to-Ground Channel Over Sea Surface at C-Band With Low Airborne Altitudes," IEEE Trans. Veh. Technol., vol. 60, no. 4, May 2011, pp. 1943-1948.

[9] K. Anderson, and Coauthors, "The RED Experiment: An Assessment of Boundary Layer Effects in a Trade Winds Regime on Microwave and Infrared Propagation over the Sea" Bull. Amer. Meteor. Soc., vol. 85, Sep. 2004, pp. 1355-1365.

[10] M. Levy, "Parabolic Equation Methods for Electromagnetic Wave Propagation," Institution of Electrical Engineers, London, U.K., 2000.

[11] I. Sirkova, "Brief review on PE method application to propagation channel modeling in sea environment" Cent. Eur. J. Eng., vol. 2, issue 1, 2012, pp. 19-38.

[12] Users Manual for Advanced Refractive Effects Prediction System (AREPS). San Diego, CA: Space and Naval Warfare Systems Center, 2004, pp. 17.

[13] O. Ozgun, G. Apaydin, M. Kuzuoglu, L. Sevgi, "PETOOL: MATLABbased one-way and two-way split-step parabolic equation tool for radiowave propagation over variable terrain", Computer Physics Communications, vol. 182, issue 12, Dec. 2011, pp. 2638-2654.

[14] G. D. Dockery, R. S. Awadallah, D .E. Freund, J. Z. Gehman, M. H. Newkirk, "An Overview of Recent Advances for the TEMPER Radar Propagation Model," IEEE Radar Conference, Apr. 2007, pp. 896-905.

[15] R. Akbarpour, A. R. Webster, "Ray-tracing and parabolic equation methods in the modeling of a tropospheric microwave link," IEEE Trans. Antennas Propagat., vol. 53, no. 11, Nov. 2005, pp. 3785-3791.

Ergin Dinc [S'12] (edinc@ku.edu.tr) received his B.Sc. degree in Electrical and Electronics Engineering from Bogazici University, Istanbul, Turkey, in 2012. He is currently a research assistant at Next-Generation and Wireless Communications Laboratory (NWCL) and pursuing his Ph.D. degree at the Electrical and Electronics Engineering Department, Koc University, Istanbul, Turkey. His current research interests include communication theory, beyondLine-of-Sight (b-LoS) communications with troposcatter and atmospheric ducts.

Ozgur B. Akan [M00-SM07] (akan@ku.edu.tr) received his Ph.D. degree in electrical and computer engineering from the Broadband and Wireless Networking Laboratory, School of Electrical and Computer Engineering, Georgia Institute of Technology in 2004. He is currently a full professor with the Department of Electrical and Electronics Engineering, Koc University and the director of the Next-generation and Wireless Communications Laboratory. His current research interests are in wireless communications, nano-scale and molecular communications, and information theory. He is an Associate Editor of the IEEE Transactions on Communications, the IEEE Transactions on Vehicular Technology, the International Journal of Communication Systems (Wiley), the Nano Communication Networks Journal (Elsevier), and the European Transactions on Technology. 